\title{
Electrocardiographic abnormalities with quinine and artesunate combinations in comparison to quinine or artesunate alone in severe falciparum malaria
}

\author{
Anjali Tarai*, Kali Prasad Pattnaik, Kumar Haraprasad Mishra
}

Department of Pharmacology, PRM Medical College and Hospital, Baripada Mayurbhanj, Affiliated to North Odisha University, Odisha, India

Received: 04 July 2020

Revised: 01 September 2020

Accepted: 02 September 2020

*Correspondence:

Dr. Anjali Tarai,

Email: dr.anjalitarai@gmail.com

Copyright: ( ) the author(s), publisher and licensee Medip Academy. This is an open-access article distributed under the terms of the Creative Commons Attribution Non-Commercial License, which permits unrestricted non-commercial use, distribution, and reproduction in any medium, provided the original work is properly cited.

\begin{abstract}
Background: In Multi drug resistant falciparum malaria anti-malarial combinations are frequently used i.e. Quinine and IV Artesunate. Quinine is associated with electrocardiographic disturbances. Artesunate in high dose produce QTC prolongation in animal models, so the electrocardiogram (ECG) is thoroughly studied.

Methods: Severe falciparum malaria cases 15 to 60 years were randomly allocated into 3 treatment regimens i.e. Artesunate, Quinine alone and their combination. Electrocardiographic recordings were taken periodically in 3 groups and compared statistically.

Results: The mean QTC interval is significantly prolonged in combination treatment group from $0.40 \pm 0.02$ to $0.49 \pm 0.09(\mathrm{P}<0.05)$ ECG disturbance $(44 \%)$. QTC prolongation was commonest (i.e. $27 \%)$ with electrolyte imbalance could produce life threatening cardiac arrhythmia (Polymorphic VT with $\mathrm{Sr} \mathrm{K} \mathrm{K}^{+} 2.9$ ). Artesunate alone was list prone (i.e. only 6\%) Quinine though has comparatively more (i.e. 25\%) but there is no life-threatening cardiac arrhythmia in artesunate and Quinine.
\end{abstract}

Keywords: Artesunate, Electrocardiogram, Quinine

\section{INTRODUCTION}

Multi drug resistant falciparum Is a major threat in tropical countries. ${ }^{1}$ Recently emergence of multidrug resistance in plasmodium falciparum parasites has been found both in invitro and in vivo studies against chloroquine pyrimethamin-sulfadoxin, mefloquine and also to Quinine on the western border of Thailand. ${ }^{2}$ Therefore Various combinations of Antimalarial drugs i.e. particularly artesunate and mefloquine, artemether with lumefantrine, quinine with doxycycline, quinine and clindamycin are being recommended by various authors. ${ }^{1,2}$
Severe falciparum malaria has high mortality even with adequate treatment i.e. $(12-15 \%)$ mortality with Artesunate (15-22\%) and with Quinine. ${ }^{1}$ Quinine was earlier considered as the first line drug for this condition; however, it is associated with life threatening complications i.e. hypotension, hypoglycaemia, electrocardiographic disturbances. ${ }^{2}$ Artesunate is a potent alternative antimalarial in this condition with a better margin of safety but higher incidence of recrudescence. There is a strong theoretical background for combining artesunate and quinine because they have different mechanism of actions i.e. artesunate kills parasite by generation of carbon centered free radicals, whereas quinine inhibits haem polymerization; Again, artesunate has a broader stage specific killing both young and 
mature forms of parasites, whereas quinine is less effective against young rings. So, combining both of them may be synergistic in severe falciparum malaria. So, artemisinin and quinine were frequently combined in severe falciparum malaria at various centers with hope of better survival. But in contrast two other factors suggests that combination may not be beneficial i.e. (I) very fast parasite clearance resulting from artesunate may not be accelerated by the slower acting quinine, (II) in comparison to artesunate, quinine has a high frequency of adverse effects and both quinine and artesunate has potential for electrocardiographic disturbances like QTc prolongation so the addition of quinine with artesunate may increase the adverse effects. Though quinine rarely causes cardiovascular complications unless therapeutic plasma concentrations are exceeded, severe hypotension is predictable when the drug is administered too rapidly by the intravenous route. ${ }^{3}$ Acute over dose also may cause serious and even fatal cardiac dysrhythmias, such as sinus arrest, Junctional arrhythmia, A-V block, ventricular tachycardia and fibrillation. ${ }^{4}$ Artemisinin derivatives given up to seven days at therapeutic doses are safe in human beings..$^{5}$ But high doses may be associated with prolongation of QT interval in experimental animals. ${ }^{6,7}$ However there is yet no significant published reports on combination of artesunate and quinine combination in severe falciparum malaria particularly in patients of multi organ dysfunction syndrome. One comparative study between artesunate and quinine, IV artesunate. ${ }^{8}$ In which mainly uncomplicated $\mathrm{p}$. falciparum patients were there with few cases of complicated malaria, had concluded that combination may not be beneficial. There are also no published reports of such combination therapy from India. So, the present work was taken up to study the relative efficacy and safety of artesunate and quinine combination in comparison to artesunate or quinine alone in patients of severe falciparum malaria (mostly multi organ dysfunction syndrome) with an aim to find out the most preferred treatment option for this condition. ${ }^{9}$

Aim and objectives of the study were report of effect of quinine and artemisinin on cardiac conduction, the present work is undertaken to study ECG abnormalities with (quinine and artesunate) combinations in comparison to quinine or artesunate alone in severe falciparum malaria.

\section{METHODS}

This study was conducted in Medicine Department of S.C.B. Medical College and Hospital, Cuttack, Orissa, India.

\section{Study design}

This is a comparative, open label, prospective observational study conducted over a period from September 2003 to February 2006.

\section{Selection of patients}

\section{Inclusion criteria}

Patients within the age group of 15 to 65 years of either sex, presenting with clinical or laboratory evidence of severe falciparum malaria.

\section{Exclusion criteria}

Patients with other co-existing disorders i.e. chronic renal failure, valvular heart diseases, malignancy etc

\section{Details of drug administration}

Group I received artesunate alone as injection artesunate $2.4 \mathrm{mg} / \mathrm{kg}$ IV start. Then $1.2 \mathrm{mg} / \mathrm{kg}$ after $6 \mathrm{hrs}$. followed by $1.2 \mathrm{mg} / \mathrm{kg} /$ day $\mathrm{X}$ Subsequent 6 days for a total duration of 7 days. ${ }^{10}$

If the patient becomes conscious and able to be discharged before 7 days then given $2 \mathrm{mg} / \mathrm{kg}$ oral tab in two divided doses instead of injection up to 7 days.

Group II received quinine alone as quinine $10 \mathrm{mg} / \mathrm{kg}$ in $10 \%$ Dextrose drip given over 4 hrs. three times a day till the patient is conscious. Followed by $10 \mathrm{mg} / \mathrm{kg}$ orally 8 hourly up to 7 days along with doxycycline $100 \mathrm{mg}$ twice daily X 7 days (orally / through Ryles tube).

Group III received artesunate with quinine combination. Artesunate dose as in group-I and Quinine dose as in group-II for 7 days.

\section{ECG parameters studied}

- QTc interval- (Corrected QT interval)

- $\mathrm{QTc}=\frac{Q-T(Q T I n t e r v a l)}{\sqrt{R R(\text { RRINTERVAL })}}$ The normal range of QTc is $0.39 \mathrm{sec}+0.04$ QTc> $0.43 \mathrm{sec}$ was regarded as QT prolongation.

- $\quad$ PR Interval from beginning of P wave to beginning of $\mathrm{R}$ wave if $>002 \mathrm{sec}$ is regarded PR prolongation.

- Any other cardiac arrhythmias or electrocardiographic disturbances during drug therapy were also recorded. ${ }^{11,12}$

- Electrocardiographic disturbances- admission ECG within normal limit but found to have electrocardiographic disturbances during treatment were regarded as electrocardiographic disturbances. ${ }^{13}$

\section{Study protocol}

ECG-recordings were taken Pre drug, 1/2-1 hr, 1-2 hr, then 4-6 hrly up to $48 \mathrm{hrs}$. after initiation of ART and/or QST Drip, then 6 hrly up to completion of ART and OR/ QST, At the time if any clinically / monitor detectable cardiac arrhythmias during period of drug therapy. The last recording was taken 24 hrs. after the completion of last dose of ART and QST. 
Other investigations like Serum $\mathrm{Na}+\mathrm{K}+$ were done routinely. Routine Hemogram, Blood MP, rapid malaria tests (ICT / Optimal) etc. serum-urea / creatinine, blood sugar and LFT were also done routinely.

Specialized investigation like 2D ECHO and CT scan of the brain were done in patients with life threatening cardiac arrhythmias to rule out any organic heart or brain disease

\section{Statistical analysis}

Statistical analysis methods applied were one-way ANOVA, Chi Square test with Yates continuity correction by using 2/2 (comparing between artesunate (AT) and artesunate and quinine combination (AQ). Again, comparing between artesunate and quinine and Fisher's exact tests for comparing between number of Statistical analysis was done by microsoft. ${ }^{14,15}$

\section{RESULTS}

It was a prospective open level randomized study in which totally 90 patients of severe falciparum malaria were initially randomly enrolled into the three treatment regimens. Because of three drop outs ultimately 87 patients completed the study i.e. artesunate alone 30 patient, artesunate and quinine combination 29 patients and quinine alone 28 patients.

The mean age in the three treatment regimens were $34.57 \pm 12.56, \quad 33.21 \pm 12.03$ and $32.64 \pm 11.26$ years respectively without any significant difference $(\mathrm{P}>0.05)$. Maximum number of patient (72 to $78 \%$ ) were young in the age group of 15 to 44 years and 61 to $78 \%$ of patients were male showing a male predominance. All the patients in study were critically ill having one or more of the features of severe falciparum malaria i.e. unarousable coma (cerebral malaria), renal failure, jaundice, severe anaemia and multi organ dysfunction syndrome. The features of severe falciparum malaria were equally distributed in the three treatment groups (Table 4 and 5). Multi organ dysfunction syndrome (MODS) was the predominant manifestations in study and was present in $66 \%$ with artesunate treatment, $65 \%$ in artesunate and quinine group and $61 \%$ in quinine treatment group without significant difference $(\mathrm{P}>0.05) .{ }^{16,17}$

The primary end point of this study was assessment of mortality rate and serious drug related adverse events (i.e. cardiovascular, neurological, metabolic). The secondary end points were comparison between fever clearance time, coma recovery time and parasite clearance time.

This study showed that the use of artesunate alone in complicated falciparum malaria was associated with least number of mortality (i.e. 13\%) in comparison to $25 \%$ mortality with quinine and still higher (i.e. $31 \%$ ) mortality with quinine + artesunate combination (Table 1 and 2). But the difference was not statistically significant ( $p>0.05$ ), however if the sample size would be doubled it would have been statistically significant.

There was no significant difference between the fever clearance time, coma recovery time and parasite clearance time between artesunate alone and artesunate + quinine combination. However, the fever clearance time with artesunate and quinine was significantly earlier than quinine alone and coma recovery time was earliest with artesunate alone with significant difference from quinine.

Analysis of ECG in three treatment groups revealed that treatment with combination of artesunate and quinine was associated with maximum incidence of electrocardiographic disturbances i.e. $44 \%$ in comparison to $25 \%$ with quinine and only $6 \%$ with artesunate alone, which was significantly lower than the above two groups $(\mathrm{p}<0.05)$.

Table 1 shows that there was no significant difference in base line QTc in patients admitted with severe malaria and enrolled into three different treatment regimens.

This table 2 showed that the mean QTC interval is significantly prolonged in artesunate and quinine combination treatment group from $0.40 \pm 0.02$ to $0.49 \pm 0.09$ during therapy $(\mathrm{p}<0.05)$. But there was no significant change in the mean QTc interval after treatment with either artesunate or quinine alone.

It is evident from table 3 shows that artesunate + quinine treatment was associated with maximum number of electrocardiographic disturbances (44\%), of which QTc prolongation was commonest (i.e.27\%) and when associated with electrolyte imbalance could produce life threatening cardiac arrythmia (Polymorphic VT with $\mathrm{Sr}$ $\mathrm{K}^{+}$2.9). Whereas artesunate alone treatment group was list prone for electrocardiographic disturbances (i.e. only $6 \%)$ and even with electrolyte imbalance there was no life threatening cardiac arrythmia (only QTC prolongation with $\mathrm{Sr} \mathrm{K}^{+} 2.9$ ).

Table 1: Corrected QTc interval before drug administration in patients enlisted for three different treatment regimens.

\begin{tabular}{|lllllll|}
\hline $\begin{array}{l}\text { Different Lab. } \\
\text { Parameters }\end{array}$ & \multicolumn{2}{l}{ Patients enlisted into different treatment regimen } & F & P value & Sig. \\
\hline $\begin{array}{l}\text { QTc in seconds + SD } \\
\text { (Pre-Drug) }\end{array}$ & $0.39+0.03$ & $0.40+0.02$ & $0.38+0.03$ & 0.13 & $>0.05$ & NS \\
\hline
\end{tabular}


Table 2: Pre-drug and post drug QTc interval comparative analysis between 3 treatment regimens.

\begin{tabular}{|lllll|}
\hline Diff. treatment regimen & Pre-drug & Post drug & P value & Sig \\
\hline ART & $0.39 \pm 0.03$ & $0.40 \pm 0.06$ & $>0.05$ & NS \\
\hline ART+QN & $0.40 \pm 0.02$ & $0.49 \pm 0.09$ & $<0.05$ & Sig. \\
\hline QN & $0.38 \pm 0.03$ & $0.43 \pm 0.09$ & $>0.05$ & NS \\
\hline
\end{tabular}

Table 3: Electrocardiographic disturbances with three treatments.

\begin{tabular}{|c|c|c|c|}
\hline \multirow{2}{*}{$\begin{array}{l}\text { Electrocardio-graphic } \\
\text { disturbances }\end{array}$} & \multicolumn{3}{|c|}{$\begin{array}{l}\text { No. of patients developed electrocardiographic disturbances with Different treatment } \\
\text { Regimens }\end{array}$} \\
\hline & $\operatorname{ART}(n=30)(\%)$ & $A R T+Q N(n=29)(\%)$ & $\begin{array}{l}\text { QN }(n=28) \\
(\%)\end{array}$ \\
\hline $\begin{array}{l}\text { Prolonged QTc } \\
>0.43 \text { Sec. }\end{array}$ & $\begin{array}{l}1(3) \\
\text { QTc-0.59, Figure } 12, \\
\text { (associated with hypokalaemia) } \\
\text { Sr K+- } 2.9 \mathrm{mEq}\end{array}$ & $\begin{array}{l}8(27) \\
\text { Figure } 3 \text { (no associated electrolyte } \\
\text { imbalance) }\end{array}$ & $5(17)$ \\
\hline $1^{\text {st }}$ degree AV block & - & 1 (3) Figure 3 & $1(3)$ \\
\hline RBBB & - & $1(3)$ & - \\
\hline U wave & Nil & 2 (6) Figure 4 & 1 \\
\hline Any other Tall T & $\begin{array}{l}1 \\
\text { (Associated hyperkalaemia) }\end{array}$ & 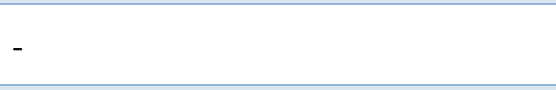 & - \\
\hline $\begin{array}{l}\text { Life Threatening } \\
\text { Cardiac arrhythmia }\end{array}$ & Nil & $\begin{array}{l}2(6) \\
\text { Case 21, Cardiac arrest Figure 3, (No } \\
\text { electrolyte imbalance), Case.2, } \\
\text { Polymorphic VT Figure } 4 \text {, (associated } \\
\text { hypokalaemia (Sr. K+2.7) }\end{array}$ & Nil \\
\hline Total & $2(6)$ & $13(44)$ & $7(25)$ \\
\hline
\end{tabular}

Table 4: Statistical analysis of electrocardiographic disturbances between three treatment regimens prolonged QTe analysis (by Chi square test).

\begin{tabular}{|c|c|c|c|}
\hline \multirow[b]{2}{*}{ Values } & \multicolumn{3}{|c|}{ Treatment regimens and No. of Patients } \\
\hline & $\begin{array}{l}\text { ART } \\
(\mathbf{n}=30)(\%)\end{array}$ & $\begin{array}{l}\text { ART+QN } \\
(n=29)(\%)\end{array}$ & $\begin{array}{l}\text { QN } \\
(n=28)(\%)\end{array}$ \\
\hline Prolonged QTc & $1(3.3)$ & $7(24)$ & $5(17)$ \\
\hline Chi Square value & & 4.248 & 3.12 \\
\hline P. Value & & $<0.05(* S)$ & $<0.05(* \mathrm{~S})$ \\
\hline
\end{tabular}

Table 5: Statistical analysis of life threatening cardiac arrythmias (by Fisher's Exact Test).

\begin{tabular}{|c|c|c|c|}
\hline \multirow[t]{2}{*}{ Values } & \multicolumn{3}{|c|}{ Treatment regimens and no. of patients } \\
\hline & $\begin{array}{l}\text { ART } \\
(n=30)(\%)\end{array}$ & $\begin{array}{l}\text { ART+QN } \\
(n=29)(\%)\end{array}$ & $\begin{array}{l}\text { QN } \\
(n=28)(\%)\end{array}$ \\
\hline Life Threatening Cardiac Arrythmias & 0 & $2(6)$ & 0 \\
\hline Fisher's Value & & 0.533 & 1.2 \\
\hline P. Value & & $>0.05(\mathrm{NS})$ & $>0.05(\mathrm{NS})$ \\
\hline
\end{tabular}

Table 6: Total No. of patients developing electrocardiographic disturbances (Statistics by Chi Square Test).

\begin{tabular}{|c|c|c|c|}
\hline \multirow[b]{2}{*}{ Values } & \multicolumn{3}{|c|}{ Treatment regimens and no. of patients } \\
\hline & $\begin{array}{l}\text { ART } \\
(n=30)(\%)\end{array}$ & $\begin{array}{l}\mathrm{ART}+\mathrm{QN} \\
(\mathrm{n}=29)(\%)\end{array}$ & $\begin{array}{l}\text { QN } \\
(n=28)(\%)\end{array}$ \\
\hline $\begin{array}{l}\text { Total no. of electrocardiographic } \\
\text { disturbances }\end{array}$ & $2(6)$ & $13(44)$ & $7(25)$ \\
\hline Chi Square & & 7.993 & 3.14 \\
\hline P. Value & & $<0.01$ (**Highly Sig) & $<0.05(*$ Sig $)$ \\
\hline
\end{tabular}


It is evidence from Table 4-6 shows that among the three treatment regimens electrocardiographic disturbances was found to be highest (44\%) in patients treated with Artesunate and Quinine combination and $25 \%$ in patients treated with Quinine alone and lowest $(6 \%)$ in patients treated with artesunate alone. In both quinine and Quinine + Artesunate treatment group the number of patients developing electrocardiographic disturbances were statistically significantly higher than no. of patients in artesunate treatment group $(\mathrm{p}<0.05$ and 0.01 respectively).

Though life threatening cardiac arrythmia was also higher $6 \%$ in artesunate and quinine treatment group in comparison to $(0 \%)$ with artesunate or quinine treatment group, but they were not statistically significant $(\mathrm{p}>0.05)$.

Table 7: Electrocardiographic disturbances and association with electrolyte imbalance in different treatment regimens.

\begin{tabular}{|llll|}
\hline & \multicolumn{3}{l|}{$\begin{array}{l}\text { Treatment regimens } \\
\text { and no. of patients }\end{array}$} \\
\hline & $\begin{array}{l}\text { ART } \\
\text { Variables }\end{array}$ & $\begin{array}{l}\text { ART } \\
\text { QN } \\
(\%)\end{array}$ & $\begin{array}{l}\text { QN } \\
(\%)\end{array}$ \\
\hline $\begin{array}{l}\text { Total no. of patients } \\
\text { developed cardiac } \\
\text { conduction disturbances. }\end{array}$ & 2 & 13 & 7 \\
\hline $\begin{array}{l}\text { No. of patients with } \\
\text { associated electrolyte } \\
\text { imbalance }\end{array}$ & 2 & $2(15)$ & $1(14)$ \\
\hline $\begin{array}{l}\text { No. of patients without } \\
\text { electrolyte imbalance }\end{array}$ & 0 & $11(85)$ & $6(86)$ \\
\hline Fisher exact test value & 0.132 & & 0.216 \\
\hline P. Value & & $\begin{array}{l}<0.05 \\
(\mathrm{~S})\end{array}$ & $\begin{array}{l}<0.05 \\
(\mathrm{~S})\end{array}$ \\
\hline
\end{tabular}

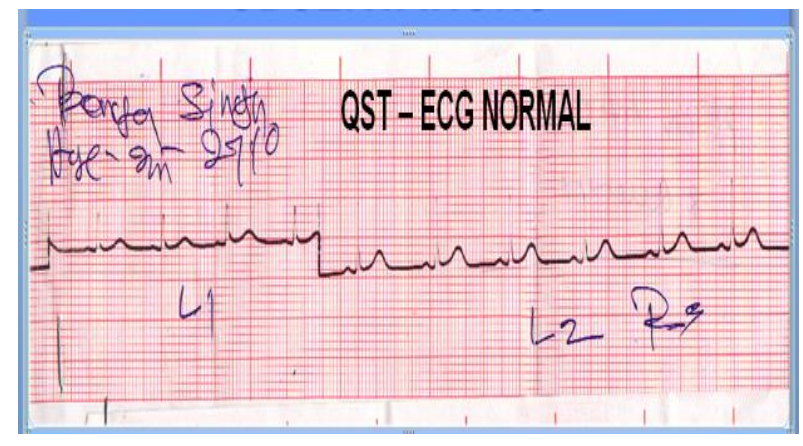

Figures 1: QST ECG normal.

Table 7 showed that $100 \%$ (2 out of 2) cases of electrocardiographic disturbances in artesunate group, $15 \%$ (2 out of 13) cases of cardiac conduction disturbances in Artesunate + Quinine group and 14\% (1 out of 7) cases of electrocardiographic disturbances in Quinine group were associated with electrolyte imbalances. Though incidence of electrocardiographic disturbances without associated electrolyte imbalance and
/ or pre-existing heart disease are negligible with artesunate alone but it is $85 \%$ with artesunate + quinine group and $86 \%$ with quinine alone treatment group which are statistically significant.

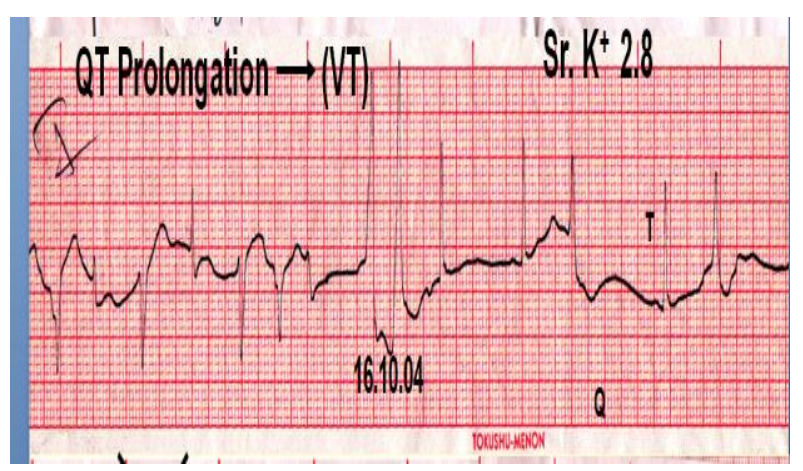

Figures 2: QT prolongation.

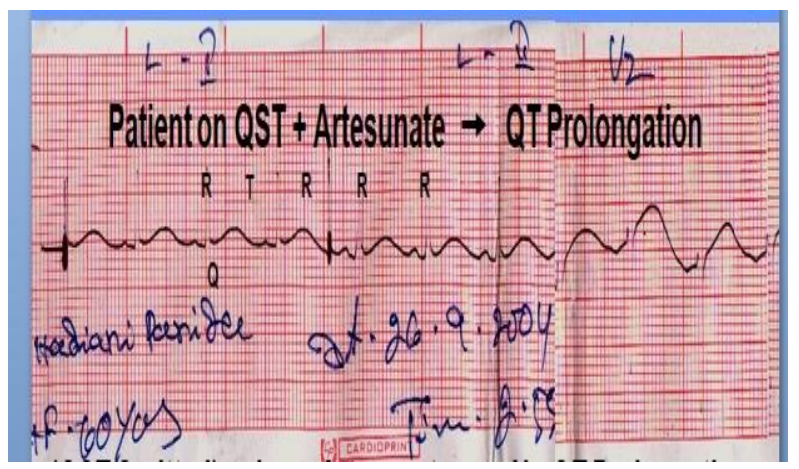

Figures 3: Patients QST+ Artesunate- QT prolongation.

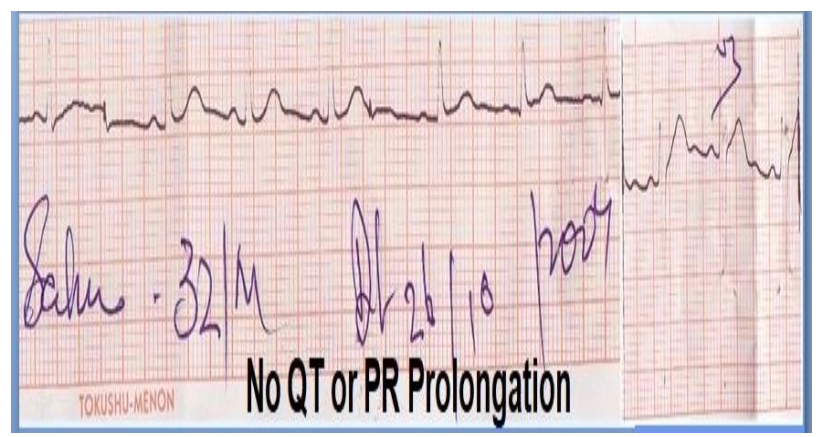

Figures 4: No QT or PR prolongation (Patient on Artesunate).

Only two cases showed QT Prolongation one associated with Hypokalaemia (Sr. K+2.9).

The various electrocardiographic disturbances found with artesunate + quinine combination was prolongation of QTc interval (24\%), first degree heart block 3\%, u wave in $6 \%$ and also life threatening cardiac arrythmias (i.e. cardiac arrest and polymorphic VT) in $6 \%$ of patients. Quinine alone was also associated with QTc prolongation (in $17 \%$ cases), first degree heart block $(3 \%) \mathrm{u}$ wave $(3 \%)$, but life threatening cardiac arrythmia was not 
found with quinine in study. QTc interval prolongation is most common electrocardiographic disturbances found in both above treatment groups which signifies that they are prone for triggered beats and ventricular tachycardia. In comparison no life-threatening cardiac arrhythmia was found in patients treated with artesunate alone. Only 2 cases $(6 \%)$ of electrocardiographic disturbances (i.e. QTc prolongation and Tall $\mathrm{T}$ ) was found with artesunate in study which was mainly associated with electrolyte imbalance.

\section{DISCUSSION}

\section{Comparative analysis of drug related adverse events}

\section{Electro cardio graphic disturbances}

Acute over dosage of quinine cause serious and fatal cardiac arrhythmia and even normal loading dose of quinine $(20 \mathrm{mg} / \mathrm{kg})$ results in significant lengthening of QTc interval. ${ }^{10,11}$ Artesunate in high dose also produce QTc prolongation in animal models though it is not common with therapeutic dose in human beings. ${ }^{11}$ So the effect of quinine or artesunate alone or their combination on ECG is thoroughly studied in this research work.

\section{Total no. of electrocardiographic disturbances (Table 7)}

This study showed that the total number of electrocardiographic disturbances was highest 44\% (13 out of 29 cases) in patients treated with artesunate and quinine combination, followed by $25 \%$ (7 out of 28 ) patients treated with quinine alone and least only $6 \%(2$ out of 30 cases) treated with artesunate alone. In comparison to artesunate alone, number of patients developing electrocardiographic disturbances was significantly higher in, artesunate and quinine combination group $(\mathrm{p}<0.01$ [highly significant] $)$ and also in quinine alone treatment group $(\mathrm{p}<0.05$ [significant]). The electrocardiographic disturbances in combination group was also higher than quinine alone, but it was not statistically significant however, if the number of patients is increased to double in both groups, there would have been a significant difference. The commonest type of electrocardiographic disturbance in study prolongation of QTC interval.

\section{Effect on QTc interval (Table 1 and 3)}

The mean QTc interval in three groups before administration of artesunate alone, artesunate and quinine or quinine alone was $0.39 \pm 0.03,0.49 \pm 0.02$ and $0.38 \pm 0.03$ seconds respectively without any significant difference between them $(p>0.05)$. The mean QTC recorded after treatment with either artesunate or quinine alone was $0.40 \pm 0.06$ and $0.43 \pm 0.09$ seconds respectively, which were statistically not significantly different from pre drug values $(p>0.05)$. But the mean QTC recorded with combination of artesunate and quinine was $0.49 \pm 0.09$ seconds, which was significantly prolonged than artesunate alone $(\mathrm{p}<0.05)$. This study report are consistent with the reports of previous study in which a significant prolongation of QTc interval occurred from a mean value of 0.42 seconds to 0.47 seconds after treatment with combination of artesunate + quinine, but no significant change in QTc occurs after artesunate treatment alone $(0.43$ second to 0.44 seconds after drug). ${ }^{12}$ In study along with analysing the change in the mean QTc interval we had also comparatively assessed the number of patients developing QTc prolongation in the three treatment regimens.

It was found that similar to other study reports of no change in mean QTc interval after artesunate treatment, in study also only $3 \%$ of patients treated with artesunate developed prolongation of QTc interval which was significantly less in comparison to QTc prolongation in $27 \%$ of patients in artesunate and quinine group and $17 \%$ of patients in quinine group alone $(\mathrm{p}<0.05)$. QTc prolongation is the commonest electrocardiographic disturbances found in patients treated with quinine and artesunate or quinine alone. But, unlike reports of prolongation of mean QTc after quinine by previous study; in study, no statistically significant prolongation of mean QTC interval was found after treatment with quinine alone. This may possibly be due to, use of loading dose of quinine $(20 \mathrm{mg} / \mathrm{kg})$ but in study dose of quinine used was $10 \mathrm{mg} / \mathrm{kg} 8 \mathrm{hrly}$, however significantly more number of patients in quinine treatment group in study developed QTc prolongation in comparison to artesunate treatment group were QTc prolongation is rare. ${ }^{12,13}$ This shows that though in animal study artesunate has been associated with QTc prolongation with high doses, but in human beings in normal therapeutic doses it is rarely associated with QTc prolongation in comparison to quinine alone and artesunate and quinine combination. ${ }^{11-13}$ The significant prolongation of mean QTc interval in artesunate and quinine group may possibly be due to additive effect of QTc prolongation potential of quinine (also found in patients) and QTc prolongation potential artesunate in animal studies.

Other electrocardiographic disturbances in three treatment regimens (Table 3-6)

The other ECG disturbances found with artesunate + quinine treatment group in study (Table 2) are $1^{\text {st }}$ degree heart block (3\%) (Figure 3) incomplete right bundle branch block (RBBB), U wave $(6 \%)$ and even life threatening cardiac arrythmias $(6 \%)$ (i.e. 2 out of 29 patients, case No.2 had polymorphic ventricular tachycardia (Figure 1) and case No. 21 developed cardiac arrest. Out of this two-life threatening arrythmias, the patients who developed polymorphic VT also had associated hypokalaemia, however the case who developed cardiac arrest had neither pre-existing heart disease nor electrolyte imbalance. 
Treatment with quinine alone was also associated with prolongation of QTc interval first degree heart block 3\% and $\mathrm{U}$ wave $3 \%$ but there was no life-threatening arrhythmia, however they are less with quinine alone in comparison to combination.

Treatment with artesunate alone was associated with ECG changes in 2 cases only and in both the cases there were also associated electrolyte imbalance. One case of prolonged QTc with artesunate (Figure 4) had associated hypokalaemia and the second ECG change in artesunate group was a tall $\mathrm{T}$ wave, which was due to presence of hyperkalaemia $\left(\mathrm{Sr} . \mathrm{K}^{+}\right.$6.2). But there was no lifethreatening cardiac arrhythmia with artesunate alone. This indicates that no significant electrocardiographic disturbance occurs with use of therapeutic dose artesunate alone unless there is associated electrolyte imbalance and / or pre-existing heart disease.

\section{Life-threatening cardiac arrythmias with different treatment (Table 5)}

In this study, in artesunate and quinine treatment group there were 2 cases of life threatening cardiac arrythmias. One case (Case No.2) developed polymorphic ventricular tachycardia who had coexisting hypokalaemia and the second case (Case No.21) developed cardiac arrest who had neither pre-existing heart disease (as per ECG and ECHO study) nor had electrolyte imbalance.

But none of the 28 cases treated with quinine in study had developed life threatening cardiac arrhythmias. Similarly, none of the 30 patients receiving artesunate alone had developed life threatening cardiac arrhythmia even though two patients in that group had electrolyte imbalance. However, there was no statistically significant difference in number of life-threatening cardiac arrhythmias between the 3 treatment regimens ( $p>0.05)$.

So, study showed that electrocardiographic disturbances are significantly higher in patients treated with artesunate and quinine combination (i.e. 44\%) and quinine alone (25\%) but is least with artesunate alone (6\%), out of which prolongation of QTc interval is commonest. Life threatening cardiac arrhythmia is also found in $6 \%$ of patients treated with artesunate and quinine combination, but not found with either quinine or artesunate alone in therapeutic doses. Though both quinine and artesunate first potential for electrocardiographic disturbances no detailed study reports are available regarding ECG disturbances with combination of quinine and artesunate. In previous study have only reported significant prolongation of mean QTc interval in artesunate and quinine combination in comparison to artesunate. So ECG monitoring should routinely to be done during administration of quinine + artesunate combination. ${ }^{16,17}$ Though it is reported that myocardial conduction disturbances is very unusual with plasma concentration of quinine below $20 \mathrm{mg} / \mathrm{l}$ and acute overdose may cause fatal cardiac dysrhythmias, but in study we are unable to estimate the serum concentration of quinine. ${ }^{7,14}$ However we found electrocardiographic disturbances in $25 \%$ patients treated with quinine only, but there was no life threatening cardiac dysrhythmias. So, when facilities exist routine ECG monitoring may be considered during quinine or quinine + artesunate therapy. As artesunate is associated with least number of electrocardiographic disturbances, it may be safely administered where ECG monitoring is not available. ${ }^{18,19}$

\section{CONCLUSION}

Analysis of ECG in three groups revealed in combination was prolongation of QTC interval (24\%), first degree heart block $3 \%$, u wave in $6 \%$ and also life threatening cardiac arrythmias (i.e. cardiac arrest and polymorphic VT). Quinine alone was also associated with QTC prolongation (in $17 \%$ cases), first degree heart block (3\%) $\mathrm{u}$ wave $(3 \%)$, of both the groups signifies that they are prone for triggered beats and ventricular tachycardia. In comparison with artesunate no life-threatening cardiac arrhythmia alone. Only 2 cases (6\%) of electrocardiographic disturbances (i.e. QTC prolongation and Tall T) was found with artesunate in study which was mainly associated with electrolyte imbalance.

\section{ACKNOWLEDGEMENTS}

Author would like to thanks Dr. Biranchi Narayan Mohapatra, HOD Department of Medicine, SCB Medical College, Cuttack and Dr. Mrutyunjay Behera HOD, Department of Cardiology, SCB Medical College, Cuttack.

\section{Funding: No funding sources}

Conflict of interest: None declared

Ethical approval: The study was approved by the Institutional Ethics Committee

\section{REFERENCES}

1. Newton PN, Chierakul W, Ruangveerayuth R, Silamut K, Teerapong P, Krudsood S et al. A comparison of artesunate alone with combined artesunate and quinine in the parenteral treatment of acute falciparum malaria. Trans $\mathrm{R}$ Soc Trop Med Hyg. 2001;95(5):519-25.

2. Newton PN, Chierakul W, Ruangveerayuth R, Silamut K, Teerapong $\mathrm{P}$, Krudsood S, et al. A comparison of artesunate alone with combined artesunate and quinine in the parenteral treatment of acute falciparum malaria. Trans R Soc Trop Med Hyg. 2001;95(5):519-25.

3. WHO, 2004: World Health Report. Report of the Director General WHO; 2004.

4. Artemether-Quinine Meta-analysis Study Group. A meta-analysis using individual patient data of trials comparing artemether with quinine in the treatment of severe falciparum malaria. Trans R Soc Trop Med Hyg. 2001;95(6):637-50. 
5. Krishna S, White NJ. Pharmacokinetics of quinine, chloroquine and amodiaquine. Clinical implications. Clin Pharmacokinet. 1996;30(4):263-99.

6. Goodman and Gillman's: The pharmacological Basis of Therapeutics; $10^{\text {th }}$ edn, 2004. Chapter 40; James W Tracy and Leslie T Webster; Drugs used in the chemotherapy of protozoal infections malaria.

7. Bateman DN, Dyson EH. Quinine toxicity. Adverse Drug React Acute Poisoning Rev. 1986;5:215-33

8. White NJ, Looareesuwan S, Warrell DA, Warrell MJ, Chanthavanich P, Bunnag D et al. Quinine loading dose in cerebral malaria. Am J Trop med Hyg. 1983;32(1):1-5.

9. Therapeutic drugs: $2^{\text {nd }}$ Edition 2002: Artemisinin: Edited by Colin Dollery. Churchil Living Stone. P.208-13

10. Win K, Jhan M. Comparison of combinations of parenteral artemisinin derivatives plus oral mefloquine with IV quinine plus tetracycline for treating cerebral malaria; Bulletin of the WHO. 1992;70:777-82.

11. Harrison's Principle of Internal Medicine, $16^{\text {th }} \mathrm{Edn}$, 2005. Chapter 195 by Nicholas J White, Joel G Breman Malaria and Babesiosis: Disease caused by Red Blood Cell. Parasites. Page-1218

12. Malaria: Principles and Practice of Malariology. Vol.2. edited by Walther H, Wernsdorfer and Sir Ian Migregor. Churchill Living Stone, 1988.

13. China Co-operative Research Group on quinghaosu and its derivatives as antimalarias; The Chemistry and synthesis of quinghaosu derivatives. J Traditional Chinese Med. 1982;2:9-16.

14. Martin Bland. An Introduction to Medical Statistics, Oxford Medical Publications, edn 3,1987.

15. ames E. De Muth. Basic statistics and Pharmaceutical statistical application. Publisher, Dekan Inc. New York, 1999; 821.

16. Bateman DN, Dyson EH. Quinine toxicity. Adverse Drug React Acute Poisoning Rev. 1986;5:215-33.

17. China Co-operative Research Group on quinghaosu and its derivatives as antimalarias 1982(c) Antimalarial efficacy and mode of action of quinghaosu and its derivatives in experimental models. Journal of Traditional Chinese Medicine.

18. Devries PJ and Dien TK: Clinical pharmacology \& therapeutic potential of artemisinin and its derivatives in the treatment of malaria. Drugs, 1996;52:818-36.

19. International Artemisinin study group-Artesunate combinations for treatment of malaria, metaanalysis, Lancet. 2004;363:9-17.

Cite this article as: Tarai A, Pattnaik KP, Mishra $\mathrm{KH}$. Electrocardiographic abnormalities with quinine and artesunate combinations in comparison to quinine or artesunate alone in severe falciparum malaria. Int J Basic Clin Pharmacol 2020;9:1520-7. 